\title{
A Wearable Augmented Reality System for Navigation Using Positioning Infrastructures and a Pedometer
}

\author{
Ryuhei TENMOKU Masayuki KANBARA Naokazu YOKOYA \\ Nara Institute of Science and Technology \\ 8916-5 Takayama, Ikoma, Nara, 630-0101 Japan \\ \{ryuhei-t, kanbara, yokoya\}@is.aist-nara.ac.jp
}

\begin{abstract}
This paper describes a wearable annotation overlay system using augmented reality $(A R)$ techniques. To realize $A R$ systems, the position and orientation of user's viewpoint should be obtained in real time. The proposed system measures the orientation of user's viewpoint by an inertial sensor and the user's position using positioning infrastructures in environments and a pedometer. The system specifies the user's position using the position ID received from RFID tags or IrDA markers which are the components of positioning infrastructures. When the user goes away from them, the user's position is alternatively estimated by using a pedometer.
\end{abstract}

\section{Introduction}

Since computers have made remarkable progress in resent years, a wearable computer can be realized[1]. At the same time, Augmented Reality (AR) which merges the real and virtual worlds has received a great deal of attention as a new method for displaying information[2]. If we construct an annotation overlay system for real scene using AR techniques with a wearable computer, it can be applied for many applications[3].

To realize wearable AR systems, the position and orientation of user's viewpoint are needed for acquiring the relationship between the real and virtual coordinate systems. Usually, Global Positioning System (GPS) is used outdoors, a magnetic sensor is used indoors to measure the user's position, respectively. Since each sensor can not be used both indoors and outdoors, if we construct a wearable AR system which can be used indoors and outdoors, we must combine positioning methods for outdoor and indoor environments. However, there remain some problems such that the hardware configuration of the system is complicated and the system needs to switch the sensors between indoors and outdoors. Therefore, we propose a wearable AR system which overlays annotations on real scene images to guide the user indoors and outdoors seamlessly.

\section{Annotation Overlay System on a Wearable Computer}

AR systems which overlay annotations on the real scene need three kind of information: a position and an orientation of the user's viewpoint, and an annotation database of information associated with the real world. The system obtains the orientation of the user's viewpoint using an inertial sensor which is attached to the user's headset. The annotation database is stored in a wearable computer.

The proposed system measures the user's position indoors and outdoors using positioning infrastructures and a pedometer. The system identifies the user's accurate position using positioning infrastructures when the user is near the components of positioning infrastructures. In this paper, RFID tags and IrDA markers are used as components of positioning infrastructures. Both of them record position IDs, and the proposed system can specify the user's position by receiving position IDs with sensors which are equipped by the user. When the user goes away from components of positioning infrastructures, the user's position is estimated by using a pedometer. The pedometer consists of an electronic compass and acceleration sensors. The former can detect which direction the user walks toward. The latter can count how many steps the user walks. These two data and the user's pace make it possible to estimate the user's position in the neighborhood of components of positioning infrastructures.

\section{Hardware Configuration of Prototype Sys- tem}

Figure 1 shows a hardware configuration and data flow of the proposed wearable AR navigation system. The user equips five sensors, a notebook PC and a display device. Five sensors can obtain the position and orientation of the user's viewpoint and the real scene image. These data are sent to the notebook PC. The notebook PC generates annotation overlay images from these data and a database of annotations which is stored in the PC. The user can see annotation overlay images through the display device which is 


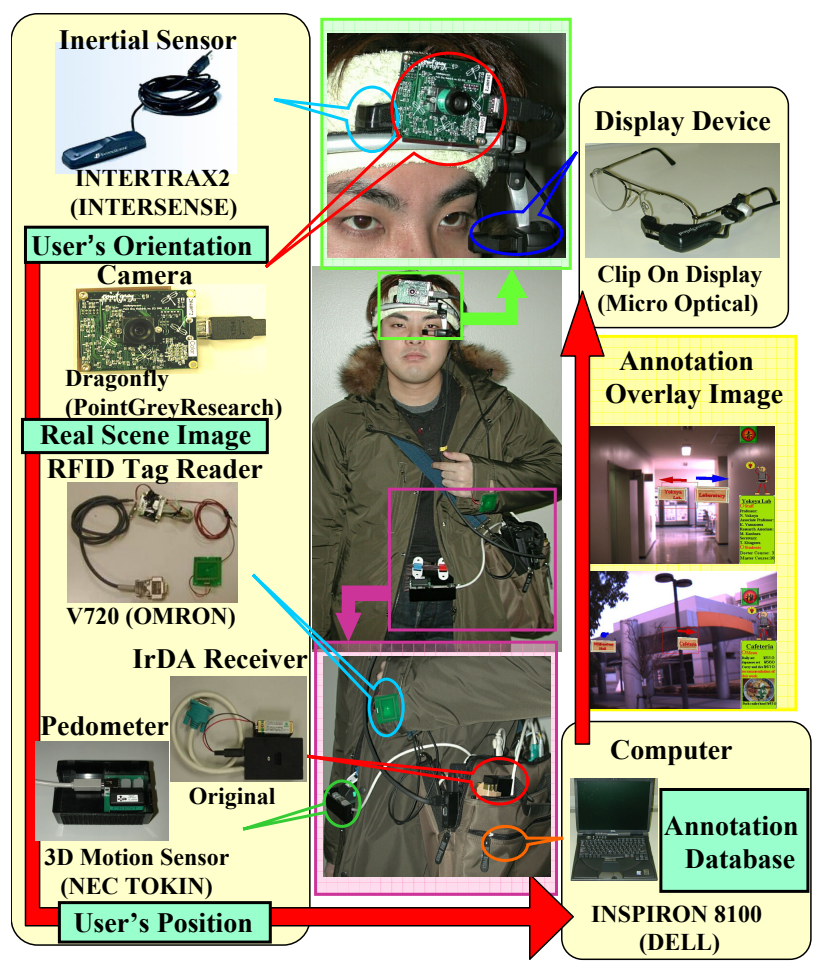

Figure 1. Hardware configuration of the proposed system.

attached to the user's headset. Components of the proposed system are described in more detail in the following.

Sensors The user equips the following five sensors. The data obtained with them is transmitted to the computer through USB, IEEE1394, or serial connection.

Inertial sensor (Intersense: InterTrax ${ }^{2}$ ) An inertial sensor is attached to the user's glasses and measures the orientation of the user's viewpoint.

Camera (Point Grey Research: Dragonfly) A camera is attached to the user's headset and captures the real scene image from the user's viewpoint. It can capture RGB 24bit color image of $640 \times 480$ pixels.

IrDA receiver (Original) An IrDA receiver is attached to the user's bag as shown in Figure 1. The IrDA receiver receives infrared ray including position IDs.

RFID tag reader (OMRON: V720) An RFID tag reader is attached to the user's wrist as shown in Figure 1. The RFID tag reader reads the position IDs recorded in RFID tags.

Pedometer (NEC TOKIN: 3D motion sensor) A pedometer can measure pitch, roll, and yaw. It can also measure accelerations in the horizontal directions.

Computer (DELL: Inspiron8100, PentiumIII 1.2GHz, 512 Mbytes memory) A computer is carried in the user's shoulder bag. It holds a database of annotation information for generating annotation overlay images.

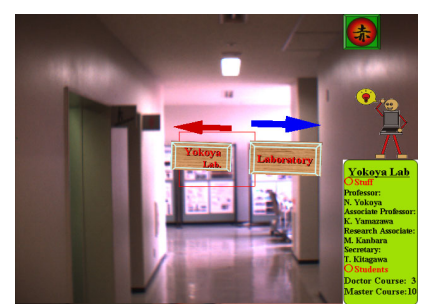

(a)

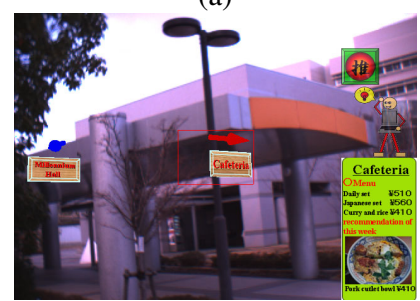

(c)

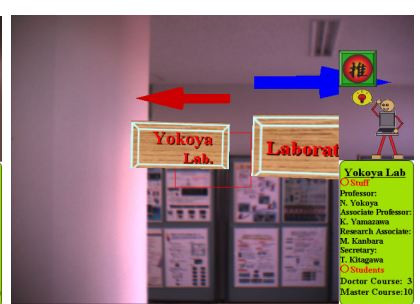

(b)

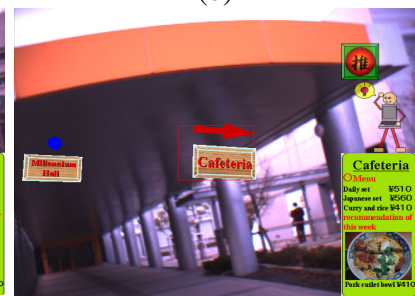

(d)
Figure 2. Annotation overlay images.

Display device (MicroOptical: Clip On Display) A display device is a video see-through display device. It is attached to headset as shown in Figure 1. It can present a $640 \times 480$ color image to the user.

\section{Experiment and Concludion}

We have carried out an experiment using the proposed wearable AR system in our campus. Figure 2 (a) and (c) show the annotation overlay images when the system identifies the user's position using positioning infrastructures, and (b) and (d) in Figure 2 are ones at the user's position estimated with the pedometer when the user walks some steps from components of positioning infrastructures.

We have proposed the annotation overlay system on a wearable computer using positioning infrastructures and a pedometer. We have also shown that the proposed system can be used both indoors and outdoors seamlessly through the experiment.

\section{Acknowledgments}

This research is partially supported by Core Research for Evolutional Science and Technology (CREST) Program "Advanced Media Technology for Everyday Living" of Japan Science and Technology Corporation (JST) and also by the Grant-in-Aid for Scientific Research from the Ministry of Education, Culture, Sports, Science and Technology (MEXT).

\section{References}

[1] S. Mann: "Wearable Computing: A First Step Toward Personal Imaging, ” IEEE Computer, Vol. 30, No. 2, pp. 25-32, 2002.

[2] R. Azuma: "A Survey of Augmented Reality, " Presence, Vol. 6, No. 4, pp. 355-385, 1997.

[3] R. Tenmoku, M. Kanbara, and N. Yokoya: "A wearable augmented reality system using an IrDA device and a passometer," Proc. SPIE, Vol. 5006, pp. 478-486, 2003. 